\title{
Compressive fatigue behavior and its influence on the thermoelectric properties of p-type $\mathrm{Bi}_{0.5} \mathrm{Sb}_{1.5} \mathrm{Te}_{3}$ alloys
}

Yun Zheng,,$\dot{t}$ Qiang Zhang, " Xianli Su, ${ }^{+}$Xinfeng Tang $\dot{t *}$

$\dagger$ Key Laboratory of Optoelectronic Chemical Materials and Devices, Ministry of Education, Jianghan University, Wuhan 430056, China

* State Key Laboratory of Advanced Technology for Materials Synthesis and Processing, Wuhan University of Technology, Wuhan 430070, China

॥ Key Laboratory of Interface Science and Engineering in Advanced Materials, Ministry of Education, Taiyuan University of Technology, Taiyuan 030024, China Corresponding author: tangxf@whut.edu.cn 

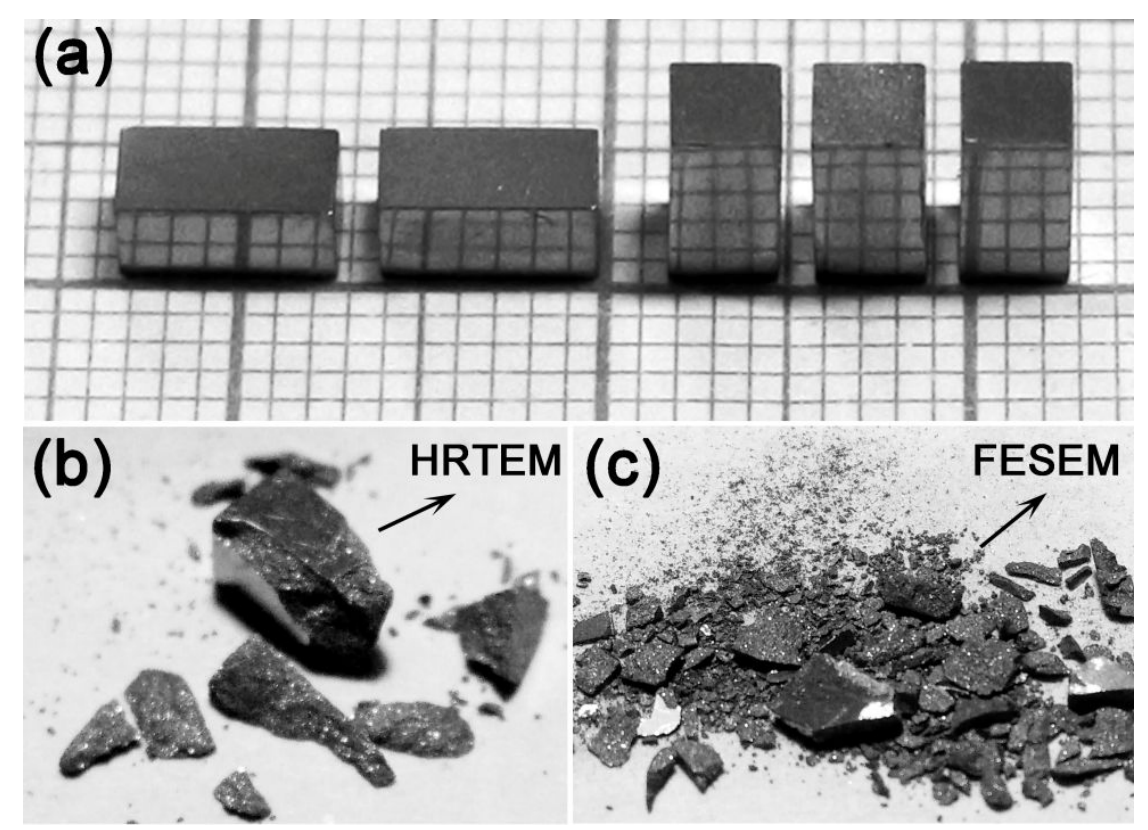

Figure $\mathrm{S1}$. The p-type $\mathrm{Bi}_{0.5} \mathrm{Sb}_{1.5} \mathrm{Te}_{3}$ pillars (nominal dimension: $6 \mathrm{~mm} \times 3 \mathrm{~mm} \times 3 \mathrm{~mm}$ ) (a) before and (b)-(c) after compressive fatigue failure. The large pieces in (b) and (c) were used for HRTEM and FESEM analyses, respectively. 


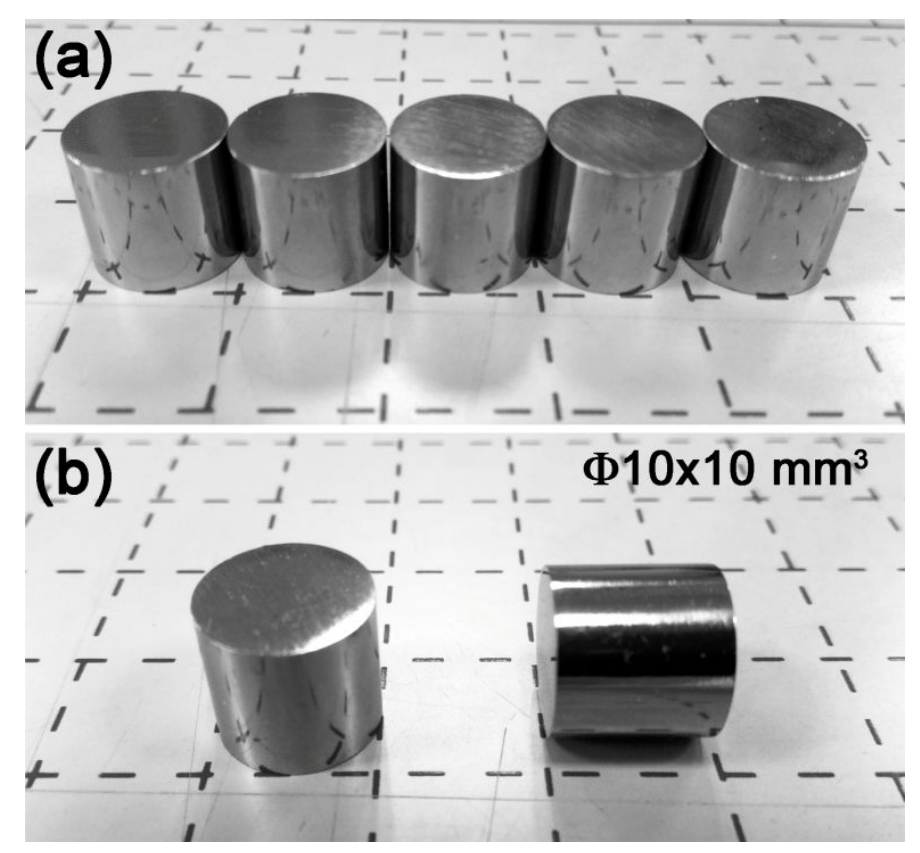

Figure S2. The cylinders (nominal dimension: $\Phi 10 \mathrm{~mm} \times 10 \mathrm{~mm}$ ) prepared for thermoelectric properties test after fatigue experiment. 

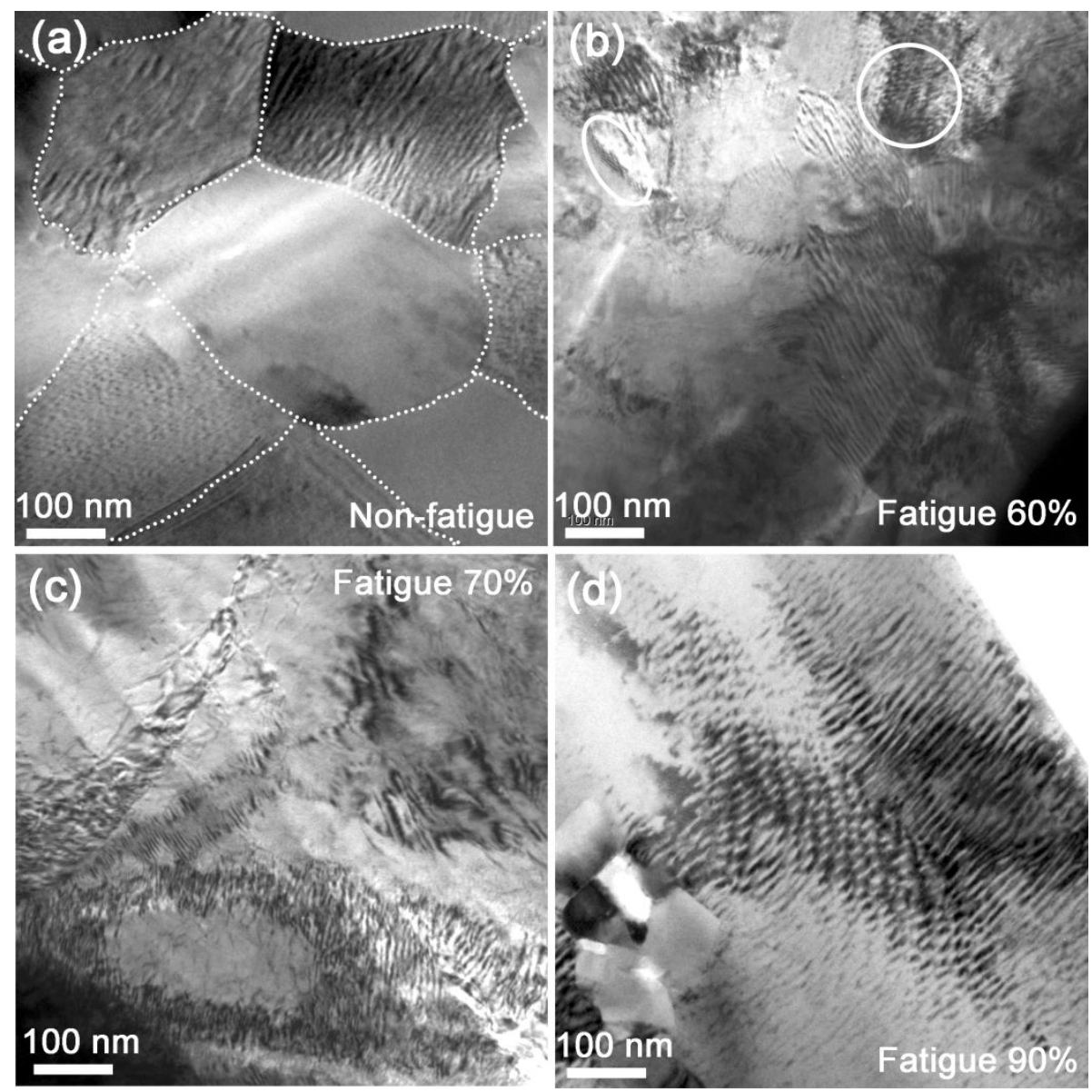

Figure S3. The HRTEM images of (a) non-fatigue samples and fatigue specimens subjected to the cyclic stress ratios of (b) $60 \%$, (c) $70 \%$ and (d) $90 \%$. 

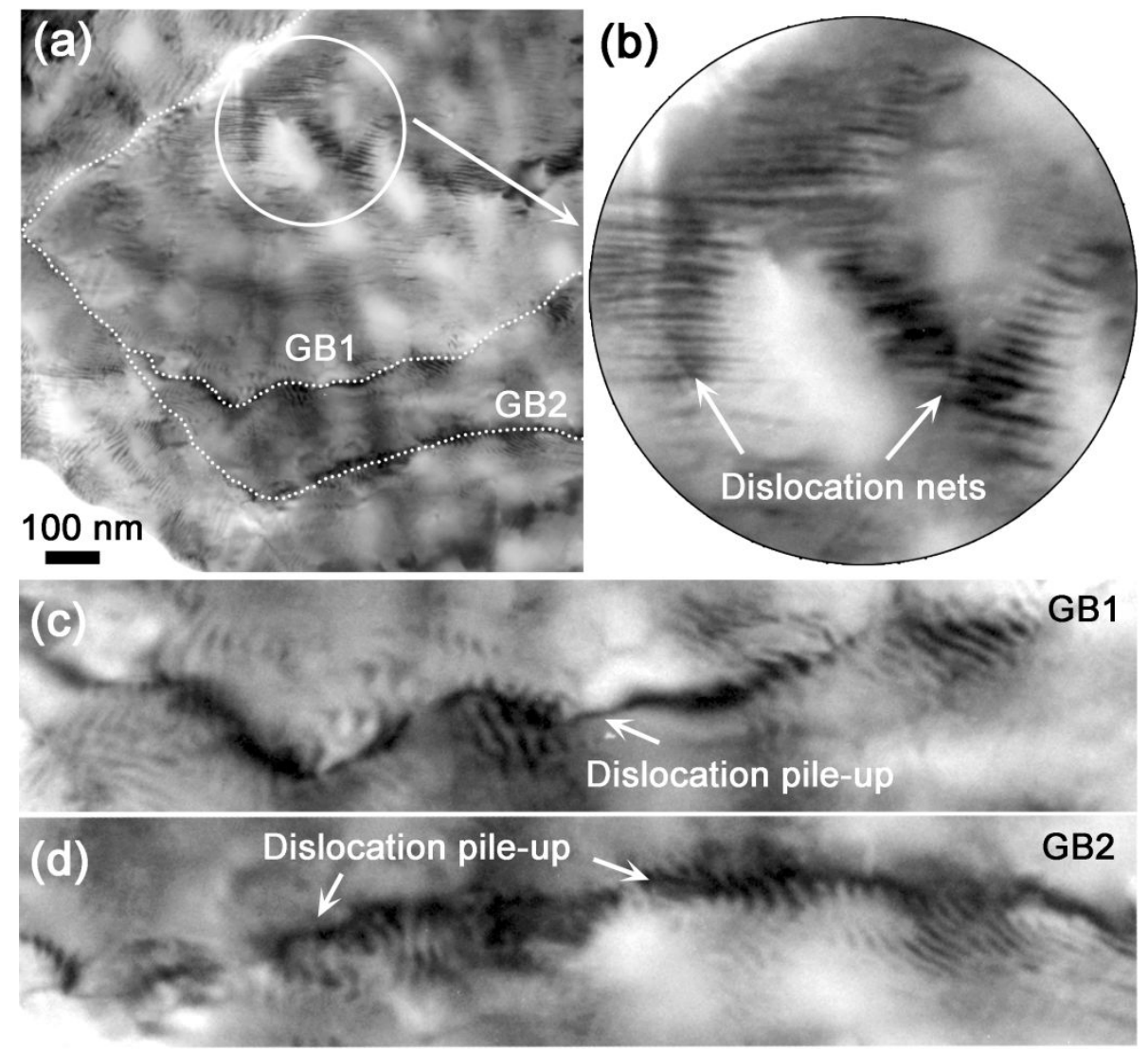

Figure S4. The HRTEM images of p-type $\mathrm{Bi}_{0.5} \mathrm{Sb}_{1.5} \mathrm{Te}_{3}$ samples after cyclic compressive failure with the stress ratio of $70 \%$, (a) the dash lines indicate the grain boundaries (GB), (b) the enlarged view of the circular region in (a). (c) and (d) correspond to the enlarged areas of GB1 and GB2, respectively. Dislocation pile-up can be clearly seen at the grain boundaries. 